\title{
BMJ Open Medical students' experience of the hidden curriculum around primary care careers: a qualitative exploration of reflective diaries
}

\author{
Ravi Parekh (D) , ${ }^{1}$ Melvyn Mark Jones (D) , ${ }^{2}$ Surinder Singh, ${ }^{2}$ Jack Shi Jie Yuan, ${ }^{3}$ \\ See Chai Carol Chan, ${ }^{4}$ Saniya Mediratta, ${ }^{5}$ Rhys Smith, ${ }^{6}$ Elinor Gunning, ${ }^{1}$ \\ Camille Gajria, ${ }^{1}$ Sonia Kumar, ${ }^{1}$ Sophie Park ${ }^{2}$
}

To cite: Parekh R, Jones MM, Singh S, et al. Medical students' experience of the hidden curriculum around primary care careers: a qualitative exploration of reflective diaries. BMJ Open 2021;11:e049825. doi:10.1136/ bmjopen-2021-049825

- Prepublication history for this paper is available online To view these files, please visit the journal online (http://dx.doi org/10.1136/bmjopen-2021 049825).

Received 03 February 2021 Accepted 06 July 2021

\section{Check for updates}

(c) Author(s) (or their employer(s)) 2021. Re-use permitted under CC BY-NC. No commercial re-use. See rights and permissions. Published by BMJ.

${ }^{1}$ Medical Education Innovation \& Research Centre, Department of Primary Care \& Public Health, Imperial College London, London, UK

${ }^{2}$ Research Department of Primary Care and Population Health, UCL, London, UK ${ }^{3}$ St George's Healthcare NHS Trust, London, UK ${ }^{4}$ Ipswich Hospital NHS Trust, Colchester, UK

${ }^{5}$ Addenbrooke's Hospital, Cambridge, UK

${ }^{6}$ Buckinghamshire Hospitals NHS Trust, Amersham, UK

Correspondence to

Ravi Parekh;

r.parekh@imperial.ac.uk

\section{ABSTRACT}

Objectives Primary healthcare internationally is facing a workforce crisis with fewer junior doctors choosing general practice (GP) as a career. In the UK, a national report on GP careers highlighted adverse influences during medical school on students' career choices. The authors explored these influences in two urban UK medical schools, both with relatively low numbers of students entering GP training.

Design Using a phenomenological approach, the authors thematically analysed the reflective diaries of four medical students who were recruited as 'participant researchers' over a period of 10 months. These students made regular reflexive notes about their experiences related to GP career perceptions in their academic and personal environments, aiming to capture both positive and negative perceptions of GP careers. The research team discussed emerging data and iteratively explored and developed themes.

\section{Setting Two UK medical schools}

Participants Undergraduate medical students Results Seven key themes were identified: the lack of visibility and physicality of GP work, the lack of aspirational GP role models, students' perceptions of a GP career as default, the performativity of student career choice with the perceptions of success linked to specialism, societal perceptions of GP careers, gender stereotyping of career choices and the student perception of life as a GP.

\section{Conclusions Students overwhelmingly reflected} on negative cues to GP careers, particularly through their experience of the hidden curriculum. Three recommendations are made: the need for increased representation of GP role models in clinical curricula content delivery and senior leadership; ensuring GP clerkships involve an active and authentic student role with patients, enabling students to experience GP's 'work' including managing complexity, uncertainty and risk. Finally, institutions need to consider students' experiences of the hidden curriculum and the effect this can have on students' perception of careers, alongside the challenges of rankings and perceived hierarchical positioning of disciplines.
Strengths and limitations of this study

- The study uses a longitudinal design with a phenomenological approach to gain an accurate sense of the medical students' experiences of the hidden curriculum related to general practice careers.

- Students were themselves 'participant researchers' who were involved in the analysis of the data to authentically understand and interpret their reflections.

- A limitation is the low number of participants, however this allowed us to have engaged participants able to continue data collection over a longitudinal period.

- Furthermore, the results are not necessarily generalisable as two institutions were involved who are both urban and research intensive.

\section{INTRODUCTION}

The WHO regards universal primary care (PC) as a central pillar to improving global health, ${ }^{1}$ and tackling COVID-19. ${ }^{2}$ Despite this growing global need for general practitioners (GPs), recruitment into GP remains an international challenge. ${ }^{3-5}$ Studies have explored the factors that may influence doctors' declining interest for careers in GP. ${ }^{67}$ These include factors such as perceived lack of intellectual challenge, social status, medical school exposure, lack of role models, working conditions and remuneration. ${ }^{5}$

In the UK, the Department of Health has set a target for $50 \%$ of medical graduates to enter GP as a career. ${ }^{8}$ However, UK medical schools are currently far from achieving this target. $^{9}$ A national report, examining how medical students can be supported towards a career in GP, highlighted the role the hidden curriculum can play in shaping career choices. ${ }^{10}$

The hidden curriculum, is the implicit academic, social or cultural message 
experienced by students and, in the context of career choice, this can include the role of bad-mouthing ('bashing') of specialties and other 'informal' comments at medical school. ${ }^{11}{ }^{12}$ These subtle but powerful messages often influence career decisions and operate at institutional levels. ${ }^{13} 14$ Previous studies looking at recruitment into GP have used focus groups, interviews and student surveys. ${ }^{12}{ }^{15}$ Although useful, these methods provide a snapshot of students' experiences and the data can suffer from recall bias. When investigating the experience of medical students, it can be the throw-away comments or non-verbal gestures, such as the subtle raising of an eyebrow, which communicate powerful messages to learners. Such communication, and certainly the context, can help to explain the lived experiences of medical students, especially in relation to decisions about GP careers.

Given this gap in the literature, we have designed this study using a phenomenological approach to answer the following questions: What are medical students' experiences of the hidden curriculum around GP careers in UK medical schools? What other factors are affecting medical students' perceptions of GP as a career?

\section{METHODS}

\section{Conceptual stance and study design}

This was a phenomenological study using reflections on clinical practice recorded in diaries by medical students recruited as 'participant researchers'. We used a phenomenological lens which assumes that reality is understood through an individual's interpretation of their experience. ${ }^{16}$ In the context of this study, the focus is on the students' experiences in medical school, which includes the explicit and hidden social, cultural and academic experiences around GP careers. However, it is important to consider how these experiences have been understood and interpreted by the individual students involved. To facilitate this conceptual approach, reflective student diaries were used; this method enables a rich understanding of experiences and has been used previously to explore sensitive areas. ${ }^{1718}$

\section{Setting}

This research was conducted in two urban UK medical schools, which were selected opportunistically, and both had low percentage of students entering GP training in national rankings. ${ }^{9}$ It was hypothesised that these students may experience more pronounced cultural practices that push students away from GP.

\section{Recruitment}

Four medical students (two male and two female) from two sites were recruited as primary data collectors, and acted as 'participant researchers'. ${ }^{19}$ These four students, in their final 2 years at medical school, had expressed an interest in undertaking educational research having already noticed the effects of the hidden curricula on their own career choices. The students have no personal connections or relationships outside of the curriculum with the researchers. None had firmly decided on their career routes, however, all four students indicated they were unlikely to pursue GP careers. Given the longitudinal nature of the study, four students were included to gather sufficient data entries over 10 months to gain a sense throughout an academic year of the medical students' experiences. The research question required participants to be able to engage over the academic year and commit to regular diary entries, therefore it was felt to be more appropriate to recruit a smaller number of students who were confident they can commit to the study.

\section{Data collection}

Prior to starting, guidance was provided to the students regarding the process of writing a reflective diary entry. During the study, the students made regular reflective entries, while attending routine educational and clinical activities, about their experiences of how a GP career was viewed by clinicians, faculty and peers. The pre-eminent feature of entries was that they were memorable and noteworthy for their content in relation to the research questions. The number of entries per student ranged from 7 to 16 entries, and the length of entries ranged from 73 words to 540 words in length.

Anonymised reflective notes were captured contemporaneously, or shortly after the event to minimise the effect of recall bias. The anonymised data in the form of wordprocessed documents were stored on an accessible drive open only to the student researchers and supervisors.

\section{Data analysis}

Given the phenomenological stance of this study, it was felt that the students themselves should analyse the data (JSJY, SCCC, SM and RS), with support and guidance provided from faculty members (RP, MMJ, SS, EG, CG, SK and SP). This was felt to be the most congruous approach, as given our epistemological stance, the students' coparticipation in analysis was important, bringing their experience and reflections into the process of data interpretation and analysis. ${ }^{20}$ The data were analysed using a thematic framework approach. ${ }^{21}$ This involves the following stages: familiarisation of the data, initial code generation, searching for themes and reviewing defining and naming these themes. ${ }^{21}$ Regular data clinics were held initially on individual sites and then jointly, to clarify the nature of the emergent data and support the students during the period of data collection and to agree on the developing themes.

No patient data were collected, and no data were collected in National Health Service (NHS) settings (hospital or PC) at either site. All data collected was fully anonymised to protect confidentiality of those who may be involved. Students consented to be participant observers. The academic researchers were not directly involved in the academic assessment of these students. 


\section{Box 1 : List of the key themes}

\section{Summary of themes identified}

Visibility and physicality of work

- Career default options

- Role modelling

Specialty hierarchy

- Societal perceptions of general practitioners (GPS)

- Gender stereotyping in medicine

GPs viewed through the student 'lens'

\section{Reflexive statement}

The project leads from both sites were all practising GPs and involved in GP undergraduate education. Contextually, this project was developed shortly after the publication of the Wass report. ${ }^{10}$ Data clinics were conducted with both faculty and students and explicit critical reflexivity was used to question and acknowledge any potential bias or assumptions during the study.

\section{Patient and public involvement}

Given the focus on assessing the hidden curriculum in medical school, patients and the public were not involved in the design, data collection or data analysis.

\section{RESULTS}

Students collected data reflecting on experiences gained from campus teaching, clinical teaching (in primary and secondary care) and the external environment (such as conversations with peers and family). Where quotes are used these represent verbatim extracts of the students' reflections, not of those informants that were observed.

The key themes developed are listed in box 1 and described in detail below with key quotes and identified by participant but not by study site, student year or gender.

\section{Visibility and physicality of work}

Students described the importance of the visibility of the work performed by doctors, and their ability to perceive its immediate rewards. They contrasted the experience in GP with acute settings. Students felt in acute settings that they were able to see the results of their actions, leading to an increased drive to learn and enjoyment.

...perhaps this is the problem with GP... it is very difficult for people to envisage the impact they have on their patients

Student 1

...being in A\&E (Emergency Medicine) and acute settings attracts medical students away from GP as you can have an easily visible impact on patients very quickly

\section{Student 1}

This observed difference in the immediacy of results between the different specialties led to the work of GPs being perceived as boring by students and faculty from other specialities:

A consultant told us we should want to do A+E surely as it is far more exciting than GP, although GP would give us a better work-life balance. Being told that you won't get any excitement out of working as a GP doesn't make that look like a viable career.

Student 2

The physicality of the specialty was also identified, specifically perceptions of dynamism related to clinicians' movement. Students noted in acute medical settings that they were able to see the physical actions of doctors standing or moving quickly; seen as actively providing care. Whereas the image of GPs passively sitting at their desk or in meetings was contrary to students' perceptions of being an active care provider. Students described how in the physically active healthcare environment it was easier for students to 'penetrate', in contrast to students' ability to feel included, sat in a GP' consulting room.

Some of the students said that they can no longer sit still for an extended period of time and implied that medicine is not about sitting around for the whole day (ie, what GPs do in the office).

Student 3

However, some students did describe the benefit of being actively involved with patient consultations in GP and the impact this can provide for patients.

actually in the GP attachment they saw so many patients and were able to help each one of them come to a solution within the 15 minute consultation.

\section{Student 3}

This perception of a lack of immediate impact, excitement and activity in GPs' work was referenced in everyday conversations. This generally passive perception of GP among students and hospital faculty feeds into the hidden curriculum of GP being seen as 'boring' with little active involvement or impact.

\section{The 'default' option}

Students often viewed GP as the 'default' career option. Our data demonstrate two main factors that shape this perception. First, students commented on the impact of being told during teaching sessions how many will become GPs, and the way in which faculty members described GP as a career, including the words, tone and insinuation used.

We all disliked the fact that throughout our respective courses, we often had the ' $50 \%$ of you will become GPs' quote thrown at us during a lecture, albeit in an entirely professional manner, but this led us to feel that most of us would be forced down a career we disliked

Student 1 
Second, entry to GP training was perceived as having little or no competition, which itself perpetuated the idea that students would not want to enter GP training, as it went against their personal aspirations of being high achieving and competitive professionals.

A friend commented to me they would do anything, even GP...It just struck me that they consider GP to be the proverbial bottom of the rung when it comes to clinical placements and that it could actually be some sort of punishment if you were to end up in GP.

\section{Student 2}

Students commented on peer discussions where GP was consistently seen as a fallback or easier option if they were not successful in other specialties.

A student told me they don't want to be doing exams and being in a hypercompetitive environment like this all their life, they would rather just be GP. This seems to always be the option that people revert to ....General practice is still time and time again seen as being the 'easy way out'.

\section{Student 4}

\section{Role-modelling}

Another recurring theme throughout the data was the description of a lack of GP role models in visible teaching clinical positions at medical school.

One of the big things that struck me was people saying I want to be like Dr X... or such and such a lecturer. ... as most of our teachers don't come from a GP background many students don't seem to want to follow that as a career.

Student 2

In addition, when GPs were involved in teaching, it was felt that they were teaching 'soft' subjects and not 'real medicine'.

GPs teach all the softer stuff-communication, ethics and law, professionalism, social determinants of health.

Student 3

\section{'Specialty hierarchy'}

An implicit model was perceived by students of a 'specialty hierarchy', with GP seen as inferior by some hospital clinicians and subsequently, students. Our data demonstrated two main factors contributing to this 'specialty hierarchy'. First, badmouthing, attitudes and stigmatisation:

A consultant reported even if they wrote it in the letter the GP wouldn't understand. They explained they need to keep the letter as simple as possible. This seems to be a recurring theme that I see in clinics or amongst hospital doctors, where GPs are spoken of in an almost derogatory manner

Student 4
Interestingly, there were more occurrences of more subtle and passive negative attitudes towards GPs mentioned in off the cuff or throwaway comments by other faculty members, who are often seen themselves as a role model for the students.

The consultant told us that cancer markers were not useful in young women, not being specific enough, then going on to comment that it was all too often that GPs do the blood test without knowing what the result could mean.

Student 1

Second, lack of intellectual rigour was also described as a recurring comment across students, faculty, friends and family. Students often described instances where hospital doctors were using mistakes made by GPs as material for a teaching session, which perpetuated the sense that GPs were intellectually inferior.

He (hospital faculty) described a GP stopping the medication of a pregnant woman ... being the wrong thing to do. This incident made me think that GPs were not very skilled individuals, leading them to take the wrong decisions.

Student 1

Interestingly, the students both commented on how this affected their perception of GPs and also that they did not want to be seen in the same way by their colleagues if they did go into GP, even if they disagreed with the comments.

Insinuations that they're (GPs) not very smart or with it, will of course put us off going into that field. Even if we know it isn't true, who wants to be looked at or considered by other doctors as not being smart?

\section{Student 4}

It is important to note that on the rare occasions when the opposite was observed, such as consultants commenting positively about GPs and the respect they have for their GP colleagues, this had a significant impact on the students' perceptions.

I've only heard a consultant say once that GPs get a lot of the brunt of the inefficiencies in our healthcare systems-it can't be easy, we don't give them enough credit for it.

\section{Student 4}

In addition, there appeared to be an accepted culture of medical school that specialisation equates to success. The data showed how, during social interactions, students described how they perceived GP as being 'easier' and less superior due to the lack of 'specialist' knowledge.

the students were discussing that GPs cannot make significant changes and are always required to refer constantly. Therefore, during the discussion, I was given the impression that having in-depth knowledge in one specialty is superior to knowing a bit of 
everything; because no matter which specialty, you would still have the knowledge of general medicine

\section{Student 3}

\section{Societal perceptions of GPs}

Societal perception of GPs also played a role. Several observations reflected how there was an ingrained message across interactions with non-medical friends, family and society more generally that being a GP was less successful than other specialties. One of the students described how a non-medical friend made a comment which was casually laughed off:

I got asked what if you don't do well at medical school, or aren't very bright and do badly in (exams) to which one of the non-medics replied that you fail and become a GP. Everyone laughed at the comment and it was brushed aside.

\section{Student 2}

These wider societal views may shape medical students' views about GP even before they enter medical school. One of the students commented on their experience when helping during a university open day:

One of the common questions I got asked was whether introducing more GP into the curriculum could dilute the scientific nature of the course.

\section{Student 2}

The students often comment on the impact of the views of friends and family, who often include key role models for the students, in shaping their view of GP.

the experiences of medical students' friends and families matter a lot and these opinions and stories (positive/negative) can largely influence students' perspectives.

\section{Student 3}

\section{Gender stereotypes in general practice}

The data also demonstrate a persistent message during medical school that GP is seen as a specialty for women and ideal for those wanting families.

Women are told directly or indirectly that they'll probably 'end up' a GP if they want children and a family

\section{Student 3}

This feminised image of GP was described consistently in the data from comments by students in social settings, to faculty discussions and opinions from families. Often the comments were subtle, or made in the context of a different discussion, but the hidden messages remained consistent.

a male consultant commented as we get more women coming into the NHS they will want to take up the part time roles of General Practice

Student 1

\section{GPs viewed through the student lens}

There were three key areas of how GP careers were viewed through medical students' eyes: work-life balance, the isolation of working as a GP and the role GPs have with their patients.

Students consistently perceived GP as having a better work-life balance than other specialties, describing shorter training time and more flexible working hours. These views often overshadowed any other aspect of the discipline, often being the most significant factor when students were discussing choosing a career as a GP.

A specialist doctor commented about the benefits of (GP) - including high pay and good lifestyle balance

Student 3

Students perceived GP as a patient-focused specialty, but also as lonely and isolating, with individual GPs interacting only with patients, rather than as part of a team, in contrast with what they have experienced in their hospital placements where they experience team ward rounds and more social team interactions.

'I now feel like there is a relative isolation of a GP practice. Whilst you may not be working with the people all the time in the hospital, the ability to meet for lunch/grab a coffee is much greater than in General Practice

Student 1

There were conflicting views from students about the desirability of the GP's role more generally. Medical students seem to have some appreciation for the role that GPs play in patient care, however, students seemed to be divided whether this was a desirable feature.

...my experience in medical education has demonstrated to me that GPs perform a vital role in coordinating patients' care, avoiding A\&E admission

Student 1

\section{DISCUSSION AND FUTURE DIRECTIONS}

This study addressed a gap in the literature, by providing the perceptions of clinical medical students towards a possible career in GP. The conceptual stance and methodological approach used captures a candid and authentic account of medical students' experiences, enabling us to enhance our understanding of the potent hidden curriculum. $^{1322} 23$

The themes identified can broadly be categorised into three areas of influences: experiences during clerkships, experiences during medical school and influences from family and society.

Students commented on the lack of visible work in community clerkships. This can be interpreted as a lack of opportunity for the students to appreciate the 'active' cognitive processing required by GPs as they manage complex, multimorbid patients, which include weighing 
up risks and benefits and tolerating a high degree of clinical uncertainty. In contrast, the more explicit experience of managing a cardiac arrest in hospital allows the students to obviously see the actions and immediate impact of interventions. In addition, the role of the students during their clerkships needs to be examined, the data suggest that students perceive GP clerkships as being passive and boring. However, when students were able to have responsibility of seeing patients and understanding the cognitive challenges, students commented on how they gained an appreciation and respect for the discipline. This finding is important, as although previous studies have demonstrated a link between undergraduate GP exposure and career choice, the quality of such placements was found to be a key factor, with a call for more longitudinal GP placements and meaningful student roles. ${ }^{6}$

The hidden curriculum throughout medical school was pervasive in the data. Interestingly, on a number of occasions students reflected on how they disagreed with the negative comments we report, but the fear of being viewed in a negative way if they were to reveal an intention to pursue a GP career, pushed them even further from considering GP. The influence of such negative comments and the impact on career choice is aligned to similar studies in the literature. ${ }^{2425}$

Finally, the influence for students of their family and society was also strong in the data. The perception of GP as being less prestigious than other specialties often reinforced this career hidden curriculum.

The concept of the hidden curriculum has been challenged in literature, with critique around how 'hidden' this curriculum may now be and how this metaphor may itself be a barrier to change, ${ }^{26}$ as well as the need to provide a detailed description of what is meant by the term 'hidden curriculum' and concrete recommendations of how it can be addressed. ${ }^{14}$ We have provided a conceptual description of the 'hidden curriculum' in the context of this study in the methodology. In addition, what is described in this study is a systemic experience of students which influence their perceptions of GP as a career. In the academic education community, this phenomenon may no longer be 'hidden', however for our students, exposure to these attitudes remain endemic.

\section{Strengths and limitations}

The phenomenological and longitudinal approach to this study, using reflective diary entries and participant researchers are key strengths to this study. The longitudinal design enabled students to showcase a variety of different interactions to obtain their experiences of the hidden curriculum. Involving the students in the analysis of the data was vital to understand and interpret the reflections.

There are limitations to this study. The institutions involved are research intensive and so the findings are not generalisable to other universities. Participant researchers themselves may share or collude in institutional norms and may not be independent or external enough to critically evaluate the culture. We acknowledge the low number of participants researchers in the study, however, this allowed us to have engaged participants able to continue data collection regularly over a longer period. The research team included both students and faculty members, who were keen to understand how students' views of GP careers are shaped.

We also acknowledge that the experiences during medical school are one of many that together will influence decision on careers. ${ }^{7}$ However, there is little research exploring the influence of the hidden curriculum using such a novel methodology to understand the students' experiences.

\section{Recommendations for practice}

This study highlights three key implications relevant for institutions in tackling the hidden curriculum experienced by students around GP careers.

First, there is a need for GP role-models for students. This is not simply increasing the numbers of GPs working in educational institutions; but to widen the teaching of GPs in the undergraduate setting beyond traditional areas in the curriculum. Coteaching with GPs, specialists, scientists and other members of the multidisciplinary team teaching together has been shown to be an effective tool in breaking down professional barriers, and role modelling mutual respect. ${ }^{27}$

Second, there is a need to ensure that community clerkships based in PC allow students to take an active role with patient management. The literature on this is extensive and demonstrates the need for students to work authentically, seeing patients who have a need to be seen by a healthcare professional, rather than patients deemed 'suitable for students'. By doing so, students understand the knowledge, challenges and skills required in PC around uncertainty, risk and patient empowerment. Many models have been developed to help educators implement such a design, including the use of longitudinal placements. ${ }^{28}$ GPs must allow students to experience and understand the cognitive processes they are working through when seeing patients with clinical complexity, medical uncertainty and ethical challenges by making the implicit explicit.

Finally, there is an imperative need for educators to tackle the pervasive impact of the hidden curriculum around careers. There is a need for all faculty to be increasingly accountable and aware of the impact of their comments, actions and the culture created which students experience. Several studies have demonstrated the impact of the phenomena of specialty 'bashing' on specialty career choice and student morale. ${ }^{24} 2930$ A zero-tolerance attitude from senior faculty towards such behaviour; alongside a transparent, trusted and fair reporting system for students and trainees who witness unprofessional comments, behaviour or attitudes, could help to address this issue.

\section{Implications for research}

There is a need to explore how these aspects of the hidden curriculum can be addressed within medical 
schools. Studies have suggested how explicit teaching on the hidden curriculum can be a useful tool to empower student themselves to critique and challenge their own experiences. ${ }^{31}$ The recommendations outlined above including more prominent GP educator role models, and higher quality longitudinal GP placements with students taking on meaningful roles, should be piloted and robustly evaluated to understand their effect on students' perceptions of GP careers.

In addition, there is a need for studies to explore how students' experiences before university as well as external influences, may influence their perceptions of GP careers. The impact of competitive medical selection on the perception of GP as a career also needs to be explored, as there may be links between certain selection processes and career intentions. ${ }^{32}$ Crucially, there is a need to discuss these tensions and issues with our students, so they can critically evaluate and challenge these cultural norms with faculty, patients and more broadly, society.

Twitter Ravi Parekh @raviparekh86, See Chai Carol Chan @carolchancarol and Sonia Kumar @SoniaKumar100

Contributors SP, SK, RP, MMJ and SS designed the study. JSJY, SCCC, SM and RS collected data. JSJY, SCCC, SM, RS, RP, MMJ, SS, EG, CG, SK and SP analysed the data and contributed to the interpretation of the data. RP drafted the manuscript with input from MMJ and SS. All authors gave approval for the final version.

Funding This report is independent research supported by the National Institute for Health Research Applied Research Collaboration Northwest London. The views expressed in this publication are those of the author(s) and not necessarily those of the National Institute for Health Research or the Department of Health and Social Care. Award/Grant number is not applicable.

Competing interests None declared.

Patient and public involvement Patients and/or the public were not involved in the design, or conduct, or reporting or dissemination plans of this research.

Patient consent for publication Not required.

Ethics approval. Ethical approval granted by UCL Research Ethics Committee project ID 12627/002 and Imperial College Medical Education Ethics Committee 18-79.

Provenance and peer review Not commissioned; externally peer reviewed.

Data availability statement No data are available. We do not have ethical permission to share data.

Open access This is an open access article distributed in accordance with the Creative Commons Attribution Non Commercial (CC BY-NC 4.0) license, which permits others to distribute, remix, adapt, build upon this work non-commercially, and license their derivative works on different terms, provided the original work is properly cited, appropriate credit is given, any changes made indicated, and the use is non-commercial. See: http://creativecommons.org/licenses/by-nc/4.0/.

\section{ORCID iDs}

Ravi Parekh http://orcid.org/0000-0003-0219-4956

Melvyn Mark Jones http://orcid.org/0000-0002-8766-7443

\section{REFERENCES}

1 World Health Organisation. Primary health care, 2019. Available: https://www.who.int/news-room/fact-sheets/detail/primary-healthcare [Accessed 25 Aug 2020].

2 World Health Organisation. Role of primary care in the COVID-19 response, 2020. Available: https://apps.who.int/iris/bitstream/handle/ 10665/331921/Primary-care-COVID-19-eng.pdf?sequence=1\& isAllowed $=\mathrm{y}$

3 Weidner A, Davis A. Influencing medical student choice of primary care worldwide: international application of the four pillars for primary care physician workforce. Isr J Health Policy Res 2018;7:57.

4 Macinko J, Harris MJ. Brazil's family health strategy. N Engl J Med
5 Selva Olid A, Zurro AM, Villa JJ, et al. Medical students' perceptions and attitudes about family practice: a qualitative research synthesis. BMC Med Educ 2012;12:81.

6 Amin M, Chande S, Park S, et al. Do primary care placements influence career choice: what is the evidence? Educ Prim Care 2018;29:64-7.

7 Querido SJ, Vergouw D, Wigersma L, et al. Dynamics of career choice among students in undergraduate medical courses. A BEME systematic review: BEME guide No. 33. Med Teach 2016;38:18-29.

8 Department of Health U. Delivering high quality, effective, compassionate care: developing the right people with the right skills and the right values - a mandate from the government to health education England: April 2013 to March 2015, 2013. Available: https://www.gov.uk/government/uploads/system/uploads/ attachment_data/file/203332/29257_2900971_Delivering_Accessible. pdf [Accessed 19 Mar 2018].

92019 F2 career destinations survey, 2019. Available: https://healtheducat ionengland.sharepoint.com/UKFPO/Website Documentation/Forms/ Alltems.aspx?id=\%2FUKFPO\%2FWebsite Documentation\%2F6 - Resources\%2FDocuments - Forms\%2C Guidance and Reports\%2FReports\%2FF2 CDS_2019.pdf\&parent=\%2FUKFPO\% 2FWebsite Documentati [Accessed 10 Sep 2020].

10 Wass V. By choice - not by chance supporting medical students towards future careers in general practice 2016.

11 Ajaz A, David R, Brown D, et al. Bash: badmouthing, attitudes and stigmatisation in healthcare as experienced by medical students. BJPsych Bull 2016;40:97-102.

12 Erikson CE, Danish S, Jones KC, et al. The role of medical school culture in primary care career choice. Acad Med 2013;88:1919-26.

13 Lempp H, Seale C. The hidden curriculum in undergraduate medical education: qualitative study of medical students' perceptions of teaching. BMJ 2004;329:770-3.

14 Lawrence C, Mhlaba T, Stewart KA, et al. The hidden curricula of medical education: a scoping review. Acad Med 2018;93:648-56.

15 Nicholson S, Hastings AM, McKinley RK. Influences on students' career decisions concerning general practice: a focus group study. Br J Gen Pract 2016;66:e768-75.

16 Cleland JA. The qualitative orientation in medical education research. Korean J Med Educ 2017;29:61-71.

17 Travers C. Unveiling a reflective diary methodology for exploring the lived experiences of stress and coping. $J$ Vocat Behav 2011;79:204-16.

18 Morrell-Scott N. Using diaries to collect data in phenomenological research. Nurse Res 2018;25:26-9.

19 Sharpe D. Participant observation, 2020University of Toronto. Available: https://research.utoronto.ca/participant-observation [Accessed 10 Sep 2020].

20 Brown MEL, Dueñas AN. A Medical Science Educator's Guide to Selecting a Research Paradigm: Building a Basis for Better Research. Med Sci Educ 2020;30:545-53.

21 Braun V, Clarke V. Using thematic analysis in psychology. Qual Res Psychol 2006;3:77-101.

22 Laqueur T. Book: boys in white: student culture in medical school. BMJ 2002;325:721a-721.

23 Phillips SP, Clarke M. More than an education: the hidden curriculum, professional attitudes and career choice. Med Educ 2012;46:887-93.

24 Holmes D, Tumiel-Berhalter LM, Zayas LE, et al. "Bashing" of medical specialties: students' experiences and recommendations. Fam Med 2008;40:400-6.

25 Wainwright D, Harris M, Wainwright E. How does 'banter' influence trainee doctors' choice of career? A qualitative study. BMC Med Educ 2019;19.

26 MacLeod A. The hidden curriculum: is it time to re-consider the concept? Med Teach 2014;36:539-40.

27 Willey JM, Lim YS, Kwiatkowski T. Modeling integration: co-teaching basic and clinical sciences medicine in the classroom. Adv Med Educ Pract 2018;9:739-51. Volume.

28 Hudson JN, Poncelet AN, Weston KM, et al. Longitudinal integrated clerkships. Med Teach 2017;39:7-13.

29 Alberti H, Banner K, Collingwood H, et al. 'Just a GP': a mixed method study of undermining of general practice as a career choice in the UK. BMJ Open 2017;7:e018520.

30 Alston M, Cawse-Lucas J, Hughes LS, et al. The persistence of specialty disrespect: student perspectives. PRIMER 2019;3:1

31 Neve H, Collett T. Revealing the hidden curriculum to medical students : insights from threshold concept theory; 2005. https:// www.ee.ucl.ac.uk/ mflanaga/abstracts/TC14Abstract32.pdf

32 McManus IC, Woolf K, Dacre J, et al. The Academic Backbone: longitudinal continuities in educational achievement from secondary school and medical school to MRCP(UK) and the specialist register in UK medical students and doctors. BMC Med 2013;11:242. 